TITLE:

\title{
Marked differences in volume phase transitions between gel and single molecule in DNA
}

\section{$\operatorname{AUTHOR}(\mathrm{S}):$}

Mayama, H.; Nakai, T.; Takushi, E.; Tsujii, K.; Yoshikawa, Kenichi

\section{CITATION:}

Mayama, H. ... [et al]. Marked differences in volume phase transitions between gel and single molecule in DNA. Journal of Chemical Physics 2007, 127(3): 034901.

\section{ISSUE DATE:}

2007-07-21

URL:

http://hdl.handle.net/2433/45690

\section{RIGHT:}

Copyright (2007) American Institute of Physics. This article may be downloaded for personal use only. Any other use requires prior permission of the author and the American Institute of Physics. 


\title{
Marked differences in volume phase transitions between gel and single molecule in DNA
}

\author{
H. Mayama \\ Nanotechnology Research Center, Research Institute for Electronic Science, Hokkaido University, \\ Sapporo 001-0021, Japan \\ T. Nakai \\ Department of Physics, Graduate School of Science, Kyoto University, Kyoto 606-8502, Japan \\ E. Takushi \\ Department of Physics, University of The Ryukyus, Okinawa 903-0129, Japan \\ K. Tsujii \\ Nanotechnology Research Center, Research Institute for Electronic Science, Hokkaido University, \\ Sapporo 001-0021, Japan and CREST, JST, Kawaguchi, Saitama 338-8570, Japan \\ K. Yoshikawa \\ Department of Physics, Graduate School of Science, Kyoto University, Kyoto 606-8502, Japan and ICORP, \\ JST, Kawaguchi, Saitama 338-8570, Japan
}

(Received 8 March 2007; accepted 18 May 2007; published online 16 July 2007)

\begin{abstract}
Volume phase transitions of a DNA gel and a single giant DNA chain caused by spermidine ${ }^{3+}$ $\left(\mathrm{SPD}^{3+}\right)$ were investigated. The change in volume for the single DNA $\left(V / V_{0} \sim 10^{-5}\right)$ was four orders of magnitude greater than that for the DNA gel $\left(\sim 10^{-1}\right)$, while the critical SPD ${ }^{3+}$ concentration for the gel $(1.8 \mathrm{mM})$ was one order of magnitude greater than that of the single DNA $(0.12-0.25 \mathrm{mM})$ at the same $p \mathrm{H}$ 6.86. We tried to describe mean-field theories with virial expansion, which is valid for the coil-globule transition of a single polymer chain, for the volume phase transitions to explain the reason why such marked differences appeared. Considering the degree of the ordering of Kuhn segments arising from the gel network structure together with the chain length of cross-linked polymer chains, the volume phase transitions were described and then the significant differences were reproduced quantitatively. We concluded that the network structure plays a significant role in the volume phase transition of the gel. (C) 2007 American Institute of Physics.
\end{abstract}

[DOI: $10.1063 / 1.2748767]$

\section{INTRODUCTION}

Since a polymer gel usually consists of many crosslinked polymer chains (subchains), it is natural to expect that the physical characteristics of a gel would reflect the intrinsic properties of the corresponding single polymer chains. ${ }^{1-12}$ In fact, the physicochemical properties of gels have often been explained in terms of the conformation of a subchain in network. However, an essential difference exists between a gel and a single polymer chain; subchains are fixed by a network structure, whereas a single polymer chain is not fixed. Therefore, it is expected that the difference gives any different aspects between volume phase transitions in a gel and a single polymer chain. However, it is still unclear how the volume phase transition of a gel is different from that of a single polymer chain. In order to solve this problem, we must use the same polymer chain in experiments on the volume phase transition in both gel and single polymer chain. We have been currently performing single-molecule observations on the conformational transition of individual giant DNA molecular chains using a fluorescence microscopy and have revealed that this transition can be interpreted as the first-order phase transition under the criterion of Landau. ${ }^{13}$ In this study, we will present the first experimental results on volume phase transitions on a DNA gel and a single DNA molecules using spermidine ${ }^{3+}$, a trivalent cation, as a condensation agent, and then we will describe their theoretical frameworks for the volume phase transitions of a free polymer chain and a fixed polymer chain in gel network structure.

\section{EXPERIMENT}

Single-molecule observation of giant DNA molecules was carried out as follows. T4DNA (Nippon Gene, $0.1 \mu \mathrm{M}$ in nucleotides) was dispersed into a phosphate $p \mathrm{H}$ standard solution $\left(p \mathrm{H} 6.86\right.$ at $25^{\circ} \mathrm{C}$, Wako, $250 \mathrm{mM}$ potassium dihydrogenphosphate and $250 \mathrm{mM}$ disodium hydrogenphosphate), containing 0.01 $\mu \mathrm{M}$ YOYO-1 (Molecular Probes) as a fluorescence dye, 4 vol \% 2-mercaptomethanol (Nacalai Tesque) as an antioxidant, and the desired amount of spermidine trihydrochloride (Calbiochem) as a condensation agent. Fluorescence images of individual DNAs were observed by a fluorescence microscope (IX-70, Olympus) equipped with a highly sensitive silicon intensifier target (SIT) camera (Hamamatsu Photonics) and recorded on video. As a measure of the volume phase transition of individual DNA molecules, we evaluated the hydrodynamic radius $R_{h}$ of indi- 


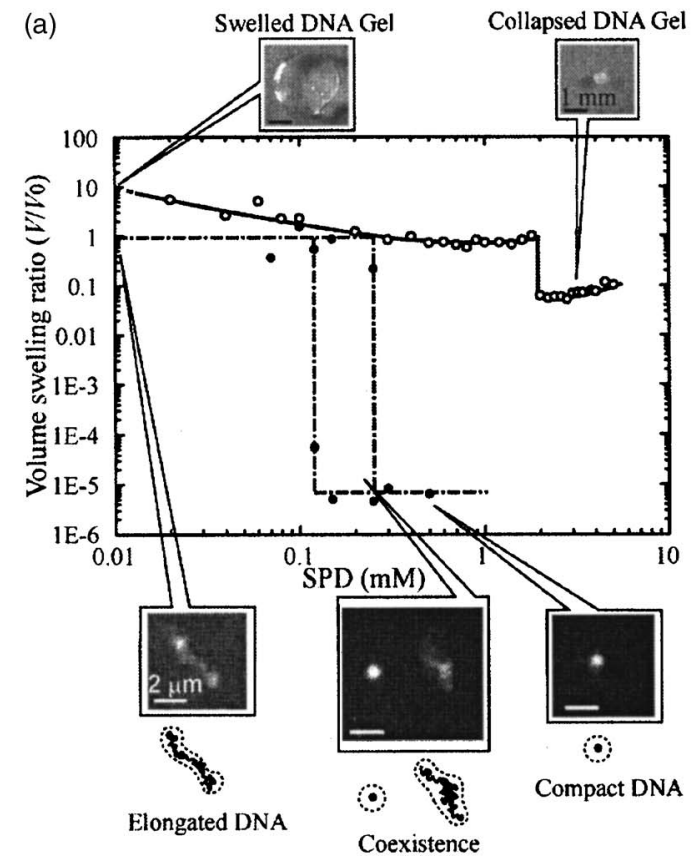

(b)
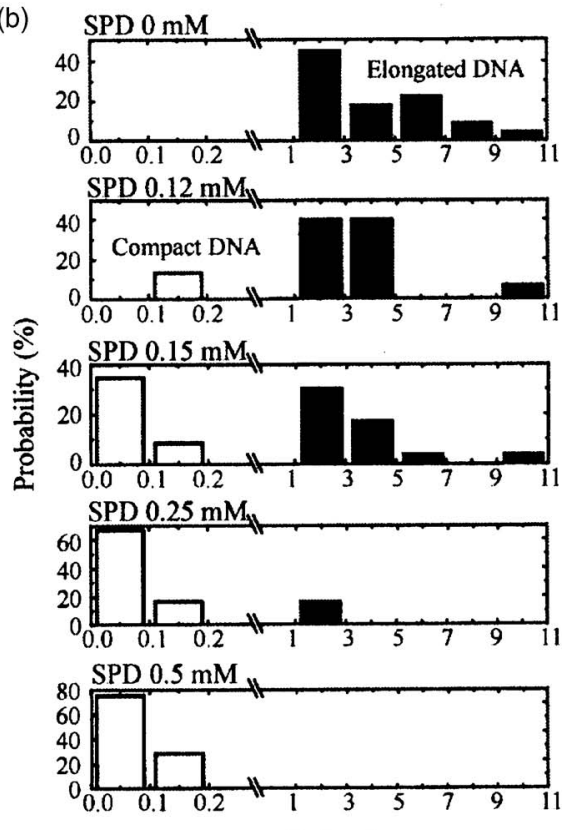

FIG. 1. (a) Volume phase transitions in a DNA gel and a single DNA molecule. Both experiments were carried out at $p \mathrm{H}=6.86$. Upper images are the swelled gel (left, $\left.\left[\mathrm{SPD}^{3+}\right]=0 \mathrm{mM}\right)$ and the collapsed gel (right, $3.2 \mathrm{mM}$ ). Lower images are fluorescence microscopic images of DNA molecules in the elongated state (left, $0 \mathrm{mM}$ ), the coexistence region (middle, $0.15 \mathrm{mM}$ ), and the compact state (right, $0.5 \mathrm{mM}$ ), with corresponding schematic representations of their conformations. (b) Distribution of the hydrodynamic radius $R_{h}$ at different $\mathrm{SPD}^{3+}$ concentrations obtained from single-DNA observation with fluorescence microscopy. vidual DNA molecules from Brownian motion observed on the video. ${ }^{14,15}$ We obtained $R_{h}$ for $15-30$ single DNA molecules under each condition. On the other hand, the experiment with double stranded DNA gel was carried out as follows. The DNA gel was prepared using $0.5 \mathrm{~g}$ DNA (Salmon tastes DNA, Sigma), $2 \mathrm{ml}$ water, and $0.25 \mathrm{ml}$ ethylene glycol diglycidyl ether (EGDE) (Aldrich) at $p \mathrm{H} 12.0$ using $0.1 \mathrm{~N}$ $\mathrm{NaOH}$ (Wako). ${ }^{5}$ The DNA gels were prepared as cylindrical gels (initial size of $\sim 1.3 \mathrm{~mm}$ in diameter and $\sim 2 \mathrm{~mm}$ in height) and placed in $10 \mathrm{ml}$ phosphate $\mathrm{pH}$ standard solution (same solutions used in single-molecule observation) containing different spermidine ${ }^{3+}\left(\mathrm{SPD}^{3+}\right)$ concentrations at room temperature $\left(22 \pm 3^{\circ} \mathrm{C}\right)$ for 3 months. The volume change was evaluated from a change in the diameter.

\section{RESULTS AND DISCUSSION}

Figure 1(a) shows the volume swelling ratio $V / V_{0}$ upon a volume phase transition in DNA gel and single DNA molecules. Individual DNA chains exhibit a large discrete transition in $0.12-0.25 \mathrm{mM} \mathrm{SPD}^{3+}$ with $V / V_{0} \sim 10^{-5}$, where the elongated and compact states coexist. The finite width of the coexistence region appears in the volume transition of a single DNA molecule because the center limit theorem cannot be attained in a single DNA chain $\left(N \ll N_{A}, N\right.$ is the number of Kuhn segment in a single DNA chain, $N \sim 560$ in a T4DNA as shown later, and $N_{A}$ Avogadro's number). ${ }^{16}$ In contrast, DNA gel exhibits a discrete transition with $V / V_{0}$ $\sim 10^{-1}$ at $1.8 \mathrm{mM} \mathrm{SPD}^{3+}$. Thus, we observed marked differences in the critical concentration of $\mathrm{SPD}^{3+}$ and in $V / V_{0}$ simultaneously. The orders of magnitude of $V / V_{0}$ in gel and single chains are consistent with those in previous studies. ${ }^{14,15}$ The typical photographs of a swelled gel $\left(\left[\mathrm{SPD}^{3+}\right]=0 \mathrm{mM}\right)$ and a collapsed gel $(3.2 \mathrm{mM})$ are shown. From the change in diameter of the cylindrical gels, $V / V_{0}$ was evaluated, where we assumed that the volume change of gel occurs homogeneously. ${ }^{5}$ On the other hand, typical fluo- rescence DNA images in elongated, coexistence, and compacted regions are also shown. Volume change of a single DNA chain was obtained from $R_{h}$, which was evaluated from the Brownian motion of individual DNA molecules under the relations $\langle R(t)-R(0)\rangle^{2}=4 D t$ and $R_{h}=k_{B} T / 6 \pi \eta_{s} D$, where $R(t)$ is the distance of the center of mass of a single DNA chain at arbitrary time $t$ from its position at $t=0, D$ is the diffusion constant, $k_{B}$ is the Boltzmann constant, $T$ is the absolute temperature, and $\eta_{s}$ is the viscosity of the solvent. Figure 1(b) shows the distribution of $R_{h}$ for single DNA chains at different $\mathrm{SPD}^{3+}$ concentrations. It is clearly shown that $R_{h}$ shows a bimodal distribution. The volume change of a single DNA molecule was evaluated from $R_{h}{ }^{3}$. Here, we determined that $V / V_{0}=1$ for the coil state of the single DNA chain and the swelling state of the DNA gel at the critical $\mathrm{SPD}^{3+}$ concentration, respectively.

As a primary cause to induce the significant difference in $V / V_{0}$, we can consider the difference of the effective contour lengths between single DNA molecule and subchains in the gel. The transition between swelled and collapsed states may be interpreted in terms of the coil-globule transition on polymer chains. It is known that the coil size $R_{c}$ and globule size $R_{g}$ with an excluded volume scale as $N^{3 / 5}$ and $N^{1 / 3}$, respectively, where $N$ is the number of Kuhn segments in a polymer chain. Since $V / V_{0}$ corresponds to $\left(R_{g} / R_{c}\right)^{3}$,

$$
V / V_{0} \approx N^{-4 / 5} \text {. }
$$

The Kuhn length of DNA is around $100 \mathrm{~nm}$. For a single T4DNA with the contour length of $56 \mu \mathrm{m},{ }^{13} N \sim 560$ and, thus, $N^{-4 / 5} \sim 10^{-2}$, while $N$ in the subchains in the gel is expected to be on the order of unity and $N^{-4 / 5} \sim 1$, at most. ${ }^{17}$ Thus, the difference in $V / V_{0}$ is attributable, at least partly, to the difference in the effective chain length between the single giant DNA and the gel.

As for the more precise discussion, we adopt a theoretical framework with mean-field treatment for the volume phase transition of single chains in order to take into account 


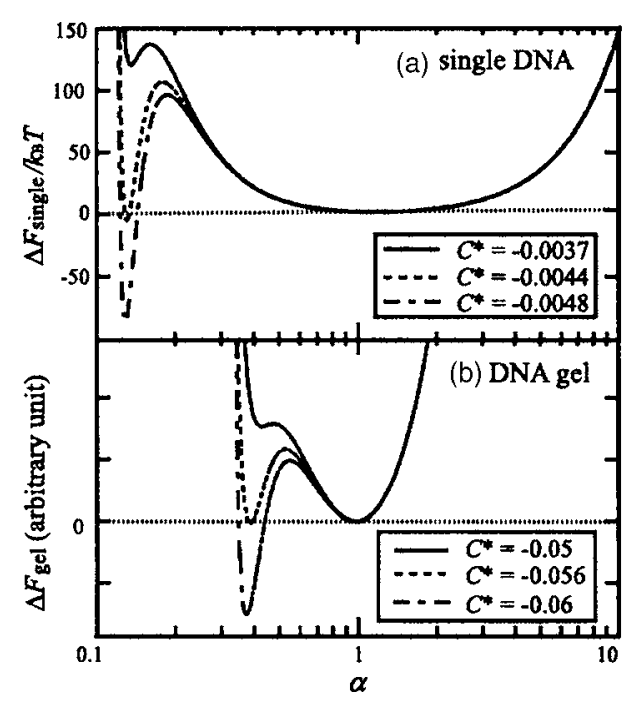

(c) single DNA

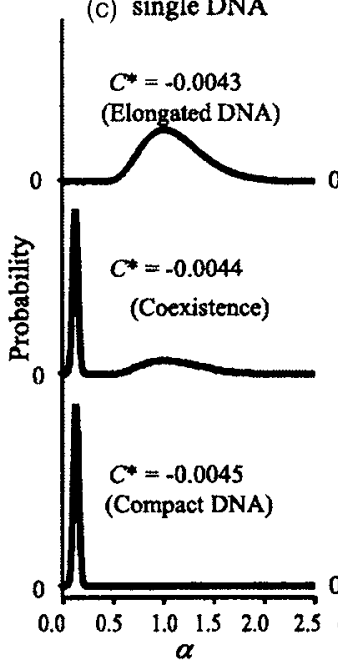

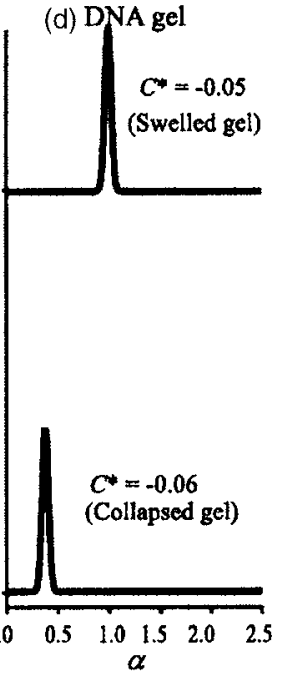

J. Chem. Phys. 127, 034901 (2007)

FIG. 2. [(a) and (b)] Free energy profiles as functions of $\alpha$ under different solvent qualities $C^{*}$ of a single polymer chain and a gel, respectively. $[(\mathrm{c})$ and $(\mathrm{d})]$. Their corresponding size distributions. Parameters are $N \sim 560$ and $\lambda=0.04$ in a polymer chain and $N_{\text {sub }} \sim 1$ and $\lambda=0.04$ in a gel. the effective chain length. On a gel, we consider the additional effect due to its heterogeneity. Such approach would be helpful for us to survey the marked difference of the volume phase transitions between the single DNA and the gel. The free energy of a single polymer chain $F_{\text {single }}$ is given as ${ }^{18}$

$$
\begin{aligned}
F_{\text {single }}[\alpha] & =F_{\text {ela }}+F_{\text {int }} \\
& \approx \frac{3}{2}\left(\alpha^{2}+\alpha^{-2}\right)+B \rho^{2} V+C \rho^{3} V+O\left(\rho^{4}\right),
\end{aligned}
$$

where $F_{\text {ela }}$ and $F_{\text {int }}$ are due to the elasticity of a polyelectrolyte chain and the interaction between Kuhn segments, respectively, $\alpha=R / R_{0}$ (the polymer chain size is $R$, the Gaussian chain size is $R_{0} \sim l_{p} N^{1 / 2}$, and $l_{p}$ is the persistence length and $l_{p} \approx 50 \mathrm{~nm}$ in DNA), $B$ and $C$ are the second and third virial coefficients, respectively, and $\rho$ is the density of the chain $(N / V)$. Considering the attractive interaction between Kuhn segments of a DNA chain, the second and third virial coefficients can be described as ${ }^{18}$

$$
\begin{aligned}
& B=B_{0}+B_{\mathrm{att}}, \quad C \approx a^{3} l_{p}^{3}, \\
& B_{0} \approx l_{p}^{2} a, \quad B_{\mathrm{att}} \approx a^{2} \sigma \tau /(\langle\sin \gamma\rangle+\lambda),
\end{aligned}
$$

where $B_{0}$ and $B_{\text {att }}$ arise from steric and attractive interactions, respectively, $a$ is the width of a chain ( $2 \mathrm{~nm}$ in DNA), $\sigma$ is the characteristic length of attractive potential, $\tau$ is the reduced temperature $\left[\tau=\left(T-T_{c}\right) / T_{c}\right], \gamma$ is the angle formed by the directors, or the main axis, of two segments $(0 \leqslant \gamma$ $\leqslant \pi / 2$ ), and $\lambda=a / l_{p}$ is the aspect ratio of the Kuhn segment. $\gamma$ is introduced for the following reason. Elongated DNAs show an intramolecular thermal fluctuation together with Brownian motion. However, when many-body collisions occur between parallel-ordered Kuhn segments $(\gamma \sim 0)$ under a suitable solution condition, a nucleus is spontaneously formed by attractive interaction between the Kuhn segments, and then it grows to form ordered structures such as a toroid and a rod. ${ }^{19-22}$ Based on this nucleation and growth process, the introduction of $\gamma$ is valid. We assumed the volume of a single DNA molecule with respect to $\gamma$ as ${ }^{18}$

$$
V \approx N a l_{p}^{2}\langle\sin \gamma+\lambda\rangle .
$$

Therefore, from $\langle\sin \gamma\rangle^{-1}=a l_{p}^{2} \rho$ at $\lambda \ll 1$,

$$
B_{\mathrm{att}} \approx a^{3} l_{p}^{2} \sigma \tau \rho
$$

We thus chose $B_{\text {att }}$ formally such that the attraction between rods is effective only at $\gamma \sim 0$. From the above discussion, the attractive term of the second virial term $B_{\text {att }} \rho^{2} V$ and the third virial term $C \rho^{3} V$ can be described as ${ }^{18}$

$$
\begin{aligned}
& B_{\mathrm{att}} \rho^{2} V=a^{3} l_{p}^{2} \sigma \tau \rho^{3} V=a^{3} l_{p}^{-4} \sigma \tau \alpha^{-6}, \\
& C \rho^{3} V=a^{3} l_{p}^{-3} \alpha^{-6},
\end{aligned}
$$

where $V=\left(\alpha R_{0}\right)^{3}$ and $R_{0}=l_{p} N^{1 / 2}$. Here, the second virial term $B_{\text {att }} \rho^{2} V$ acts as the third virial coefficients by the ordering of Kuhn segments, as shown in Eq. (7). As a result, Eq. (2) can be reduced using the effective third virial coefficient as ${ }^{18,23}$

$$
\begin{aligned}
& F_{\text {single }}[\alpha] \approx \frac{3}{2}\left(\alpha^{2}+\alpha^{-2}\right)+C^{*} \alpha^{-6}-N \ln \left[1-\frac{\lambda}{N^{1 / 2} \alpha^{3}}\right] \\
& C^{*} \approx \lambda^{3}\left(1+\sigma \tau / l_{p}\right)
\end{aligned}
$$

where $C^{*}$ is the effective third virial coefficient and corresponds to the solvent quality $\left(C^{*}>0\right.$, good; $C^{*}<0$, poor $)$ which causes the first-order phase transition. Here, we omitted the second virial term $B_{0} \rho^{2} V$ because it remains constant irrespective to the solvent quality. The last term in Eq. (9) indicates the excluded volume effect, including both second order and higher order, which avoids the anomaly of $F_{\text {single }}[\alpha] \rightarrow-\infty$ at $\alpha \rightarrow 0 .{ }^{18,23}$ Figure 2(a) shows the free energy profile of a single polymer chain obtained from Eq. (9), which indicates that the free energy profile is always bimodal with a relatively high barrier. Such characteristic profile of the free energy indicates that the folding transition of a single semiflexible polymer chain such as a long DNA should undergo a large discrete transition, i.e., a first-order phase transition under the criterion of Landau. On the other hand, the stable state with a change in $C^{*}$ can be obtained from $\partial F_{\text {single }} / \partial \alpha=0$, 


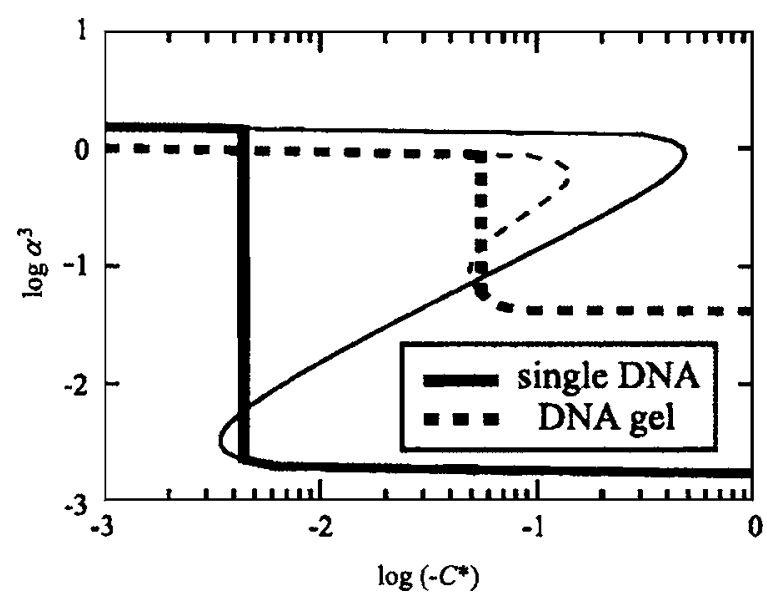

FIG. 3. Variation of the volume $\alpha^{3}$ with the solvent quality $C^{*}$ for a single DNA chain (a thick solid line) and a DNA gel (a thick dashed line) from the condition $\partial F / \partial \alpha=0$. The vertical thick lines are drawn at $C^{*}$ where the free energies of two stable states are the same.

$$
2 C^{*}=\alpha^{8}-\alpha^{4}-\frac{\lambda \alpha^{3} N^{1 / 2}}{1-\lambda N^{1 / 2} \alpha^{-3}} .
$$

Equation (11) describes a thin solid line in Fig. 3, the dependence of $\alpha$ on $C^{*}$. This type of dependence indicates that the discrete change in $\alpha$ should occur. The thick solid line depicts the manner of the discrete transition through the transition point with equal free energy minima. The volume phase transition of a single polymer chain is thus described as the large discrete transition.

On the other hand, a gel is different from a single polymer chain because the subchains are fixed in the network structure. Under the theoretical framework of a single polymer chain by taking account of the effect of the ordering of Kuhn segments, the free energy of the gel $F_{\text {get }}[\alpha]$ is given by

$$
\begin{aligned}
F_{\text {gel }}[\alpha] & =\left(F_{\text {ela }}+F_{\text {int }}\right) \\
& \approx \frac{3 N_{\text {tot }}}{2 N_{\text {sub }}}\left(\alpha^{2}+\alpha^{-2}\right)+B \rho^{2} V+C \rho^{3} V+O\left(\rho^{4}\right),
\end{aligned}
$$

where $N_{\text {tot }}$ is the total number of Kuhn segments in gel and $N_{\text {sub }}$ is the number of Kuhn segment(s) in the subchain. The elastic energy of the gel is obtained by the $N_{\text {tot }} / N_{\text {sub }}$ times of the elastic energy of a subchain.

In the virial expansion, we assume that the second virial coefficient can be expressed as

$$
B \approx B_{0}+B_{\text {att }}+B_{\text {rep }}
$$

Here $B_{0}, B_{\text {att }}$, and $C$ are essentially the same as in the case of a single polymer chain. Here, we introduced a repulsive interaction $B_{\text {rep }}$ to express the energy penalty arising from the network which disturbs the attractive interaction between Kuhn segments. $B_{\text {rep }}$ can be described as

$$
B_{\text {rep }} \approx l_{p}^{3} /\langle\sin \gamma\rangle^{2} \approx a^{2} l_{p}^{7} \rho^{2},
$$

where $\langle\sin \gamma\rangle^{-1}=a l_{p}^{2} \rho$ at $\lambda \ll 1$ and the factor $l_{p}^{3}$ in $B_{\text {rep }}$ implies the unit size of the gel network structure under the ordering of Kuhn segments at $\gamma \sim \pi / 2$. Equation (12) would be expressed using the third virial term and the contributions arising from the ordering of Kuhn segments as

$$
\begin{aligned}
F_{\text {gel }}[\alpha] \approx & \frac{3 N_{\text {tot }}}{2 N_{\text {sub }}}\left(\alpha^{2}+\alpha^{-2}\right)+\left(a^{3} l_{p}^{3}+a^{2} l_{p}^{2} \sigma \tau\right) \rho^{3} V \\
& +a^{2} l_{p}^{7} \rho^{4} V-N_{\text {tot }} \ln \left[1-\frac{\lambda}{N_{\text {sub }}^{1 / 2} \alpha^{3}}\right] .
\end{aligned}
$$

From $\rho=N_{\text {tot }} / V \approx N_{\text {tot }} /\left[\left(\alpha l_{p} N_{\text {sub }}^{1 / 2}\right)^{3} N_{\text {tot }} / N_{\text {sub }}\right] \approx 1 /\left(\alpha^{3} l_{p}^{3} N_{\text {sub }}^{1 / 2}\right)$, $F_{\text {gel }}[\alpha]$ can be reduced as

$$
\begin{aligned}
F_{\text {gel }}[\alpha] \approx & N_{\text {tot }}\left[\frac{3}{2 N_{\text {sub }}}\left(\alpha^{2}+\alpha^{-2}\right)+\frac{1}{N_{\text {sub }}} C^{*} \alpha^{-6}\right. \\
& \left.+\frac{\lambda^{2}}{N_{\text {sub }}^{1 / 2}} \alpha^{-9}-\ln \left[1-\frac{\lambda}{N_{\text {sub }}^{1 / 2} \alpha^{3}}\right]\right],
\end{aligned}
$$

where $C^{*}$ is the same to Eq. (10). Figure 2(b) shows the free energy profiles of a gel obtained from Eq. (16), exhibiting a characteristic bimodality, as in Fig. 2(a). This implies discrete characteristics on the manner of the transition. On the other hand, the stable state with a change in $C^{*}$ can be obtained as

$$
2 C^{*}=\alpha^{8}-\alpha^{4}-\frac{\lambda \alpha^{3} N_{\text {sub }}^{1 / 2}}{1-\lambda N_{\text {sub }}^{1 / 2} \alpha^{-3}}-\frac{3 \lambda^{2} \alpha^{-3}}{N_{\text {sub }}^{1 / 2}} .
$$

The thin dashed line in Fig. 3 is obtained from Eq. (17). The discrete transition point corresponds to the condition where two free energy minima are equal to each other.

Let us compare the free energy profiles of a gel and a single polymer chain in Fig. 2. In Figs. 2(a) and 2(b), the single polymer chain shows a greater discrete change in $\alpha$ between 0.12 and 1.3 with $C^{*}=-0.0044$, whereas a gel shows a smaller change in $\alpha$ between 0.39 and 1.0 with $C^{*}$ $=-0.056$. From $V \approx \alpha^{3}$, volume ratio between two states of a polymer chain (approximately three decades) is greater than that of a gel (approximately one decade). This reproduces the significant difference of $V / V_{0}$ in the experimental results. Furthermore, the magnitude of poorness $\left|C^{*}\right|$ for the transition is different. The $\left|C^{*}\right|=0.056$ of a gel is one order of magnitude greater than that $(0.0044)$ of a single polymer chain. This means that the transition of a gel occurs at a poorer solvent condition than that of a single polymer chain. Since $\left|C^{*}\right|$ is increased with increasing $\mathrm{SPD}^{3+}$ concentration in our case, this reproduces that a gel exhibits the volume transition at $1.8 \mathrm{mM} \mathrm{SPD}^{3+}$ which is one order of magnitude greater than that $\left(0.12-0.25 \mathrm{mM} \mathrm{SPD}^{3+}\right)$ of a single molecule. Figures 2(c) and 2(d) show distributions of $\alpha$ for a gel and a single polymer chain around their critical $C^{*}$, respectively. Figure 2(c) reproduces the discrete transition of single polymer chains through the coexistence region, as shown in Fig. 1(b).

Next, we would like to compare the transition manners in Fig. 3, which shows the volume phase transitions in a plot of volume $\alpha^{3}$ versus efficient solvent quality $C^{*}$. It is clearly illustrated that the magnitudes of the discreteness of $\alpha^{3}$ and $C^{*}$ at the transitions of a single polymer chain and gel are quite different on the order of decade, as discussed above. Moreover, Fig. 1(a) is thus reproduced qualitatively. Thus, the free energy descriptions within the mean-field treatment considering the chain length and the ordering of Kuhn segments reproduce the experimental results. 
In this study, we described the general theoretical frameworks with virial expansion to survey the volume phase transitions. In further study, the free energy descriptions by mixing entropy, translational entropy of counterions, etc., are required for more realistic descriptions of the volume phase transitions. Furthermore, it is very important to consider how the network structure of gel should be treated in free energy although we treated it as the energy penalty arising from the degree of the ordering of Kuhn segments.

\section{CONCLUSION}

In summary, we experimentally investigated the volume phase transitions for a gel and a single polymer chain and found the marked differences in the critical concentration of $\mathrm{SPD}^{3+}$ and $V / V_{0}$. In particular, the critical concentration of a gel was found to be one order of magnitude greater than that for a single polymer chain. Based on the theoretical framework of the coil-globule transition of a single polymer chain, the volume phase transition of a gel was described, and the significant differences in $V / V_{0}$ and critical concentration were reproduced qualitatively. It is suggested that the network structure plays a significant role in the volume transition of a gel.

\section{ACKNOWLEDGMENTS}

The authors thank Dr. S. Takagi and Dr. T. Nakagaki of the Research Institute for Electronic Science, Hokkaido University for experimental support with the processing of fluorescence images of single DNA molecules and the analysis of Brownian motion. The authors also thank Professor C.-Y. Shew of Department of Chemistry, The City University of New York and Dr. T. Iwaki of Okayama Institute for Quantum Physics for fruitful discussions.
${ }^{1}$ A. Yu. Grosberg and A. R. Khokhlov, Statistical Physics of Macromolecules (AIP, New York, 1994).

${ }^{2}$ K. Sekimoto and M. Doi, J. Phys. II 1, 1053 (1991).

${ }^{3}$ S. Katayama, Y. Hirokawa, and T. Tanaka, Macromolecules 17, 2641 (1984).

${ }^{4}$ D. Costa, P. Hansson, S. Schneider, M. Graça Miguel, and B. Lindman, Biomacromolecules 7, 1090 (2006).

${ }^{5}$ T. Amiya and T. Tanaka, Macromolecules 20, 1162 (1987).

${ }^{6}$ S. M. Mel'nikov, M. O. Khan, B. Lindman, and B. Jönsson, J. Am. Chem. Soc. 121, 1130 (1999).

${ }^{7}$ S. G. Stradoubtsev, A. A. Lyubimov, and A. R. Khokhlov, J. Phys. Chem. B 107, 12206 (2003).

${ }^{8}$ O. E. Philippova, N. L. Sitnikova, G. B. Demidovich, and A. R. Khokhlov, Macromolecules 29, 4642 (1996).

${ }^{9}$ A. R. Khokhlov and E. Yu. Kramarenko, Macromolecules 29, 681 (1996).

${ }^{10}$ P. Hansson, S. Schneider, and B. Lindman, Prog. Colloid Polym. Sci. 115, 342 (2000).

${ }^{11}$ A. Svensson, L. Piculell, B. Cabane, and P. Ilekti, J. Phys. Chem. B 106, 1013 (2002).

${ }^{12}$ S. Kudo, , M. Konno, and S. Saito, Polymer 34, 2370 (1993).

${ }^{13}$ K. Yoshikawa, M. Takahashi, V. V. Vasilevskaya, and A. R. Khokhlov, Phys. Rev. Lett. 76, 3029 (1996).

${ }^{14}$ Y. Yamasaki and K. Yoshikawa, J. Am. Chem. Soc. 119, 10573 (1997).

${ }^{15}$ K. Yoshikawa and Y. Matsuzawa, Physica D 84, 220 (1995).

${ }^{16} \mathrm{~K}$. Yoshikawa, in Complexity and Diversity, edited by E. R. Nakamura, S. Kudo, O. Yamakawa, and Y. Tamagawa (Springer-Verlag, Tokyo, 1997).

${ }^{17}$ This was based on the density measurement of a DNA xerogel prepared from the swollen state at $1.8 \mathrm{mM}$. The density of swollen DNA gel was $0.06 \mathrm{~g} \mathrm{~cm}^{-3}$, whereas the theoretical density of a cube comprised of Kuhn segments (size: $100 \times 100 \times 100 \mathrm{~nm}^{3}$ ) is $10^{-3} \mathrm{~g} \mathrm{~cm}^{-3}$. This suggests that the average length of a subchain in the prepared DNA gel is shorter than the length of a Kuhn segment. The inhomogeneity of the network structure in a gel should contribute to the distribution of subchain length.

${ }^{18}$ K. Yoshikawa and N. Yoshinaga, J. Phys.: Condens. Matter 17, S2817 (2005).

${ }^{19}$ K. Yoshikawa and Y. Matsuzawa, J. Am. Chem. Soc. 118, 929 (1996).

${ }^{20}$ T. Sakaue and K. Yoshikawa, J. Chem. Phys. 117, 6323 (2002).

${ }^{21}$ J. F. Stilck, Y. Levin, and J. J. Arenzon, J. Stat. Phys. 106, 287 (2002).

${ }^{22}$ K.-C. Lee, I. Borukhov, W. M. Gelbart, A. J. Liu, and M. J. Stevens, Phys. Rev. Lett. 93, 128101 (2004).

${ }^{23}$ T. Iwaki and K. Yoshikawa, Europhys. Lett. 68, 113 (2004). 